\title{
Epidemiology and Natural History of Non-alcoholic Fatty Liver Disease
}

\author{
Alita Mishra*, Zobair M Younossii, ${ }^{* \star \star *}$ \\ ${ }^{*}$ Center for Liver Diseases, Department of Medicine, Inova Fairfax Hospital, ${ }^{* *}$ Betty and Guy Beatty Center for \\ Integrated Research, Inova Health System, Falls Church, VA, USA
}

\begin{abstract}
Non-alcoholic fatty liver disease (NAFLD) is an important cause of liver disease burden across the world. By definition, although the histopathologic features of NAFLD are identical to that of alcoholic liver disease, its diagnosis requires absence of significant alcohol use and absence of other causes of chronic liver disease. We now know that NAFLD is not simply a disease of the Western world. It is manifested across the world, in varying rates, across gender, across varying ethnicities, and in its association with other host factors. In this review article, the definition of NAFLD, its spectrum, ranging from mild steatosis to hepatocellular injury and inflammation defined as non-alcoholic steatohepatitis (NASH) is discussed. Mild steatosis is generally a stable disease whereas NASH can be progressive. Based on current published literature, current incidence and prevalence of NAFLD and NASH are discussed. It is also accepted that these processes will continue to increase in prevalence with the rise of obesity, type II diabetes, and associated metabolic syndrome. Some of the risk factors have been well-established and are discussed. In addition, this review also presents emerging associations with other risk factors for NAFLD. Natural history of NAFLD is variable depending upon the histologic subtypes and other underlying comorbidities and is discussed in this review as well. (J Clin EXP HePATOL 2012;2:135-144)
\end{abstract}

$\mathrm{N}$ on-alcoholic fatty liver disease (NAFLD) is the most common cause of chronic liver disease (CLD) in the Western hemisphere. By some estimates, it affects $20-40 \%$ of the population in the USA. ${ }^{1}$ As the name suggests, NAFLD is a clinical entity in which patients have evidence of hepatic steatosis on imaging or histology and there is no secondary cause for the hepatic fat accumulation such as alcohol, predisposing medications or other defined liver disorders. It has been accepted that as the prevalence of obesity and metabolic syndrome rises, it will herald a parallel rise of NAFLD across the world. The common metabolic risk factors include obesity, diabetes mellitus (DM), and dyslipidemia. Management of these risk factors is extremely important to halt the progression of NAFLD.

Non-alcoholic fatty liver disease includes a spectrum of liver disorders. At one end of this spectrum is simple hepatic steatosis and the other end is non-alcoholic steatohepatitis (NASH) which is characterized by hepatocellular

Keywords: NAFLD, NASH

Received: 21.02.2012; Accepted: 07.05.2012

Address for correspondence: Zobair M Younossi, Betty and Guy Beatty Center for Integrated Research, Inova Health System and Center for Liver Diseases, Department of Medicine, Inova Fairfax Hospital, Falls Church, VA, USA

E-mail: zobair.younossi@inova.org

Abbreviations: CVD: cardiovascular disease; HCC: hepatocellular carcinoma; NAFLD: non-alcoholic fatty liver disease; NASH: non-alcoholic steatohepatitis; NHANES: National Health and Nutrition Examination Surveys; OSA: obstructive sleep apnea; PCOS: polycystic ovarian syndrome

doi: 10.1016/S0973-6883(12)60102-9 injury and inflammation, fibrosis sometimes leading to cirrhosis. Experts agree that patients with simple steatosis progress very slowly, if at all. On the other hand, NASH can be progressive, and can lead to complications such as cirrhosis and hepatocellular carcinoma (HCC). It is important to note that the histologic characteristics of NAFLD are indistinguishable from alcoholic liver disease. ${ }^{2}$ Therefore, the distinction is based on exclusion of excessive alcohol consumption. Furthermore, the diagnosis of NASH with associated fibrosis heralds a more significant prognosis as it is more likely to progress to cirrhosis with complications of hepatic failure and HCC. Non-alcoholic fatty liver disease should be suspected or considered in those who have risk factors as above of obesity, diabetes, dyslipidemia, or insulin resistance. It is generally expected to consider NAFLD in the differential diagnosis of elevated aminotransferase levels in individuals who are receiving drugs for conditions known to be associated with NAFLD. ${ }^{3}$ In fact, NAFLD should be considered as the etiology of persistent elevation of serum alanine aminotransferase (ALT) levels for which another cause cannot be found. ${ }^{3}$

\section{Incidence and Prevalence of Non-alcoholic Fatty Liver Disease and Non-alcoholic Steatohepatitis}

Non-alcoholic fatty liver disease and its various manifestations are seen in all ethnic groups, across the globe and in both genders. ${ }^{4-15}$ It is difficult to quantify the true incidence of NAFLD because of the variation in diagnosis and also due to the fact that a large number of patients may be asymptomatic. Nevertheless, a review of literature showed that the reported incidence of NAFLD varies 
wildly. An outpatient hepatology clinic in England showed an incidence rate of 29 cases per 100,000 person years. ${ }^{4}$ Two different studies from Japan have reported an incidence rate of 31 and 86 cases per 100,000 person years. ${ }^{5,6}$

An interesting study from Japan reported an annual incidence rate of $10 \%$ based on baseline examination and follow-up period of 414 days among 3147 patients. ${ }^{6}$ In this cohort, weight gain and the presence of metabolic syndrome at baseline were independent predictors of development of NAFLD. ${ }^{6,14}$ Another historical cohort study also from Japan using data from the routine health care of government employees has also shown that weight gain in those patients preceded the development of NAFLD and other features of the metabolic syndrome. ${ }^{5}$

In a study using proton magnetic resonance spectroscopy of the liver in the USA, it was shown that the frequency of hepatic steatosis varied based on ethnicity, more common in Hispanics (45\%) than in whites $(33 \%)$ and blacks (24\%). ${ }^{7}$ The higher prevalence in Hispanics mirrors the similar higher prevalence of obesity and insulin resistance in that group. However, the lower frequency of hepatic steatosis in blacks was not explained by factors such as body mass index (BMI), insulin resistance, alcohol, or medication. ${ }^{7}$ In addition, interestingly in this group, the prevalence of hepatic steatosis was greater in men than in women among whites, $42 \%$ vs $24 \%$, but this gender difference was not seen in blacks or Hispanics. ${ }^{7}$ Additionally, on liver biopsy samples of patients with NAFLD, Hispanics showed higher rates of liver injury with ballooning hepatocytes and Mallory bodies; Asians had higher rates of ballooning and lobular inflammation and African Americans were less likely to have high grades of hepatic steatosis, lobular inflammation, and fibrosis. ${ }^{8}$ A Korean study in which liver biopsies were performed on 589 consecutive potential liver transplant (LT) donors reported NAFLD prevalence of $51 \% .^{9}$ In the USA, a study of living donors for adult to adult right lobe LT candidates that were biopsied showed that $20 \%$ of them were ineligible due to $>30 \%$ steatosis on biopsy samples. ${ }^{10}$ It is accepted that the prevalence of NAFLD varies greatly based on the population studied and based on the screening test such as liver enzymes, liver histology, or imaging. ${ }^{11}$ In high-risk groups with metabolic syndrome or surrogates of diabetes, obesity, and dyslipidemia, there is a significantly higher prevalence in contrast to lower rates in unselected groups. ${ }^{11}$ Nevertheless, even in the unselected group, however, there is still a wide variation in the prevalence with reported values anywhere from $3 \%$ to $36.9 \% .{ }^{11}$

The true estimate for the prevalence of NASH is even more difficult due to the lack of histologic data and limited sensitivity of non-invasive methods for establishing the diagnosis. It is likely that NASH is greatly underdiagnosed as most affected patients are symptom-free and there is not a set criterion for routine non-invasive screening. In fact, non-invasive diagnostic testing for NASH is felt by most experts to be inadequate for diagnosing or staging the severity of this disease. ${ }^{12}$ The current gold standard in the diagnosis of NASH and NAFLD is liver biopsy which is invasive, costly, and fraught with potential sampling errors.

Historically, liver-associated enzymes have been used as surrogate markers for CLD but the problem with that is some patients have been shown to have significant liver disease in the setting of NAFLD and normal enzymes. ${ }^{13,14}$ A recently published NAFLD practice guideline states that as liver biochemistries can be within normal ranges in patients with NAFLD and NASH, they may not be sufficiently sensitive to serve as screening tests, whereas liver ultrasound is potentially more sensitive but is more expensive and cumbersome as a screening test. ${ }^{15} \mathrm{~A}$ study based on the National Health and Nutrition Examination Surveys (NHANES) using liver enzymes, conducted between 1988 and 2008 in the United States, suggested that while the prevalence of other causes chronic liver disease remained stable, the prevalence of NAFLD doubled in that time period. ${ }^{16}$ This study emphasizes the tremendous wave of liver disease-related to NAFLD that we can expect over the next few decades.

\section{Risk Factors for Non-alcoholic Fatty Liver Disease}

\section{Age}

There are many risk factors for NAFLD. Although NAFLD has been described in all ages, the prevalence of NAFLD increases with age. ${ }^{17}$ Age was noted to be in independent predictor of the development of NAFLD in Japanese women. ${ }^{6}$ In the USA, data from the third NHANES (NHANES III) showed that the peak prevalence of NAFLD was earlier in men; during the fourth decade as compared with the women with the peak in the sixth decade. ${ }^{18}$ Other studies have also shown that the prevalence of NAFLD increases with age. ${ }^{19,20}$ In addition to that, it has been shown that the likelihood of disease progression to advanced fibrosis or mortality increases in older patients with NAFLD. ${ }^{21}$ In some series, it is hard to discern whether age is truly an independent risk factors as older patients often also have higher incidence of other risk factors such as metabolic syndrome, diabetes, and obesity. There have also been studies showing that components of metabolic syndrome will increase the risk for advanced fibrosis. ${ }^{22}$ Certainly, those factors are more likely in an older age group. A study of biopsy confirmed NAFLD patients divided into older, age 60 or greater, middle-aged, ages between 50 to 60 and younger group, $<50$ done by Frith et al showed an association between the prevalence of NAFLD and fibrosis with age. ${ }^{19}$ Similarly, in a cohort of octogenarians admitted to the rehabilitation department in a geriatric hospital, the prevalence of NAFLD group of elderly patients, there was no association between NAFLD and metabolic syndrome. ${ }^{23}$ In contrast, it is important to note 
that the increase in the prevalence of NAFLD is seen in all age groups, including young children and adolescents. It goes without saying that while age appears to be a logical risk factor for NAFLD and NASH, more definitive studies are needed in this area. It may simply be a longer duration of disease manifestation or it may be a preponderance of other risk factors in the elderly that seem to show a stronger association with NAFLD and higher rate of progression to NASH and fibrosis.

\section{Gender}

More recent studies have also reported that male gender is a risk factor for fatty liver disease. ${ }^{24}$ However, there are conflicting data. The prevalence of NASH in Western population studies has suggested a female predominance of NASH, with women representing $60-83 \%$ of the diagnoses. ${ }^{25}$ In another prospective study, the incidence of NAFLD was higher in men than in women. ${ }^{6,14}$ In an Asian study, of the 26,527 subjects receiving medical care, the prevalence of NAFLD was $31 \%$ in men and $16 \%$ in women. ${ }^{26}$ In a retrospective study of 58 men and 307 women who underwent gastric bypass, the prevalence of NASH was noted to be $60.3 \%$ in men as compared with $30.9 \%{ }^{27}$ These results are interesting because given the patient population, the two genders were similar to each other in terms of other risk factors and yet in this group, lower prevalence was noted among women. In another study of 400 adults in an Army medical center in the USA utilizing ultrasound and liver biopsy, it was shown that NAFLD patients were more likely to be male (58.9\%). ${ }^{28}$ It is possible that NAFLD and it progression is different in men and women. More research is needed in this area.

\section{Ethnicity and Race}

The definitive implications of ethnicity and race as risk factors are unclear. Most likely the observed prevalence parallels the incidence of underlying metabolic syndrome and obesity as opposed to the ethnicity and race itself being a true risk factor. However, there are many unknowns in this area also. As mentioned previously, in the USA, the highest rates of NAFLD are seen in Hispanics, followed by whites and then blacks based on population studies. ${ }^{7}$ Wagenknecht et al have reported that other markers for NAFLD are similar across ethnic groups and yet Hispanics do have the highest prevalence of NAFLD, hepatic steatosis, and elevated aminotransferase levels, followed by whites with the lowest rates in blacks. ${ }^{29}$ Yet, at the same time, other markers such as triglycerides (TGs) and age are only associated with NAFLD in Hispanics. ${ }^{29}$ It is possible that these apparent ethnic and familial differences are representative of an underlying genetic susceptibility. There are reports of familial clusters of NAFLD. In a recent systematic review of NAFLD, the heritability of hepatic steatosis is reported at 0.27 based on large family-based cohorts. ${ }^{24}$
Based on genotyping data to the epidemiology of NAFLD, the most important genetic contributor is most likely a polymorphism in adiponutrin/patatin-like phospholipase-3 (PNPLA3); this encodes the I148M protein variant that has been identified to be the determinant of hepatic steatosis and serum ALT levels. ${ }^{30}$ Hispanics were noted to have the highest rate of this allele. ${ }^{30}$ Outside of the Hispanic population, in a study of 253 Italian patients with NAFLD, the PNPLA3 polymorphism was shown to be associated with both steatosis and fibrosis severity. ${ }^{31}$ The mechanism for this is believed to be due to PNPLA3 predisposing to NAFLD by interrupting TG hydrolysis within hepatocytes. ${ }^{32}$ With regards to other factors, among the Asian Indian population, based on a study of 95 men, the APOC3 C-482T and T-455C polymorphisms were shown to be associated with an increase prevalence of insulin resistance and NAFLD and it was later replicated in a nonAsian Indian population. ${ }^{33}$ Another variant of the gene PNPLA3-S4531 is associated with significantly lower liver fat content and this correlates with it being more common in blacks (0.104), and less common in whites (0.003) and Hispanics (0.008). ${ }^{30}$ Another study of 7176 individuals who completed genome-wide association study of computed tomography (CT)-detected hepatic steatosis confirms the importance of PNPLA3 variants in the degree of fat accumulation in the liver. ${ }^{34}$ There are multiple other genetic polymorphisms that have been implicated in predisposing to NAFLD or NASH and may account for the racial and ethnic variations seen in populations. More research is on-going in this area.

\section{Diabetes Mellitus Type II}

Type II DM is an important aspect of the metabolic syndrome. We have known for years that type II diabetes and fatty liver have had a close relationship. In one recent study done of patients with type II DM, it was noted that there was a $69 \%$ prevalence of NAFLD on ultrasound imaging. ${ }^{35}$ It is also important to note that NASH and advanced fibrosis are often observed in diabetic patients that are asymptomatic clinically and in some cases with normal liver enzymes. Another recent study from India showed that 127 of 204 diabetic patients displayed fatty liver on ultrasound and among those who consented to a liver biopsy and had fatty liver on ultrasound, $87 \%$ had biopsy-proven NAFLD. ${ }^{36}$ In addition, the presence and predisposition to obesity and hyper-triglyceridemia in diabetic patients further amplifies the risk for developing steatosis. It is postulated that in diabetics and also in people with insulin resistance, the rate of peripheral adipose lipolysis is increased which then leads to an influx of free fatty acids into the liver which are incorporated into hepatic TGs and hepatic steatosis. ${ }^{37}$ There is also evidence that patients with diabetes and NAFLD have higher risk of progression, cirrhosis, and mortality than NAFLD 
patients without diabetes. ${ }^{38}$ Based on the NHANES III (1988-1994) data, Stepanova et al have noted that in that cohort of 991 subjects presumed to have NAFLD, the presence of type II diabetes was in independent predictor of mortality in addition to being a significant hazard for liver-related mortality. ${ }^{39}$

\section{Elevated Cholesterol}

Elevated cholesterol or more specifically hyper-triglyceridemia is frequently associated with both obesity and type II DM and is another important aspect of the metabolic syndrome. Elevated cholesterol or hyper-triglyceridemia has been reported in $20-92 \%$ of patients with NAFLD but authors do note that most of these patients had other components of the metabolic syndrome. ${ }^{40}$ It is accepted as an established risk factor for the development of NAFLD. In a 2003 study of 282 subjects with fatty liver, using ultrasound imaging, it was noted that the prevalence of hypertriglyceridemia was 7 times more frequent than in the control group and about 3 times higher than in the group with mild steatosis. ${ }^{41}$

\section{Obesity}

It has been long established that obesity is a known risk factor for hepatic steatosis. In NAFLD, robust data come from the bariatric surgery population. Intraoperative biopsies have become routine during bariatric surgery. Based on that data, there is compelling evidence that obesity is a strong risk factor for NAFLD and NASH. Ong et al reported that over $95 \%$ of bariatric surgery patients had fatty liver, $20-30 \%$ had NASH, and $10 \%$ had advanced fibrosis. ${ }^{13}$ Depending on the group, the median prevalence of NASH in the obese population is $33 \%$, with a range from $10 \%$ to $56 \% .^{42-44,46-51}$ In addition, based on this large body of data from obese patients, the prevalence of simple steatosis ranges from $30 \%$ to $37 \% .{ }^{42,43}$ The more impressive data are that within obese patients; NAFLD ranges from $57 \%$ to $98 \%$ in nondiabetic obese patients. ${ }^{44-46}$ It is noteworthy, however, that in some patients, NAFLD is a risk factor based on presence of central obesity and not high BMI. ${ }^{52}$ Experts agree that central obesity as determined by waist-to-hip ratio is strongly associated with insulin resistance and hence even within a normal BMI, its presence in individuals counts as a criteria for metabolic syndrome and risk for NAFLD.

\section{Polycystic Ovarian Syndrome}

Recent advances in understanding of polycystic ovarian syndrome (PCOS) have established it as an entity strongly associated with insulin resistance and metabolic syndrome. It is the most common endocrine disease in the female population and affects about $10 \%$ of the female population. ${ }^{53-55}$ The main clinical feature of PCOS includes chronic anovulatory cycles, oligomenorrhea, and hirsutism. Polycystic ovarian syndrome and its association with metabolic syndrome and shared features have led to extensive research in its association with NAFLD. In a prospective study of Chilean women with PCOS, $41 \%$ were noted to have NAFLD based on ultrasonographic evidence. ${ }^{56}$ In other studies of obese PCOS patients, the incidence of NAFLD and NASH is even higher and that PCOS patients are to be considered a high-risk group for the development of NAFLD and NASH. ${ }^{57}$

\section{Obstructive Sleep Apnea}

Obstructive sleep apnea (OSA) is an increasing public health issue across the world with estimated prevalence of $1-4 \%$ of general population and $25-35 \%$ of obese individuals. ${ }^{58}$ Human studies have indicated that OSA is associated with an increase in liver enzymes, and treatment of OSA has been shown to decrease liver enzymes. ${ }^{58}$ Studies have shown that sleep apnea patients have a significantly greater amount of visceral fat and insulin resistance compared with obese controls, and indexes of sleep disordered breathing are positively correlated with visceral fat, but not with BMI or total or subcutaneous fat. ${ }^{59}$ Based on this, some experts suggest that OSA should be considered as part of the metabolic syndrome. Hypoxia, as is common in OSA patients, has been known to inhibit biogenesis and respiration of the mitochondria leading to decrease in the number and function of the mitochondria and this probably leads to insulin resistance. ${ }^{60}$ In a study of 101 patients undergoing bariatric surgery with extensive clinical and histologic data for whom polysomnography data were available, subjects with histologic NASH were found to have significantly lower lowest desaturation, lower mean nocturnal oxygen saturation, higher respiratory disturbance index, and higher ALT/aspartate aminotransferase ratio compared with non-NASH controls. ${ }^{61}$ Further studies are needed in this area to better understand but it appears that OSA most likely aggravates the development of NAFLD to NASH in obese individuals.

\section{Miscellaneous Factors Associated with Fatty Liver}

Non-alcoholic fatty liver disease has been associated with multiple other conditions such as surgical interventions, medications, and metabolic diseases. Obese patients who underwent jejunoileal bypass, once a popular weight loss surgery, were noted to be particularly prone to NAFLD and cirrhosis, such that this procedure is no longer done and reversal of the operation has been recommended. ${ }^{62}$ Rapid weight loss, total parenteral nutrition, starvation, lipoatrophy, inborn errors of metabolism, petrochemical exposures, refeeding syndrome, bacterial overgrowth, and certain pesticides have all been associated with abnormal liver-associated enzymes and NAFLD as described in the literature. ${ }^{12}$ In addition, although human 


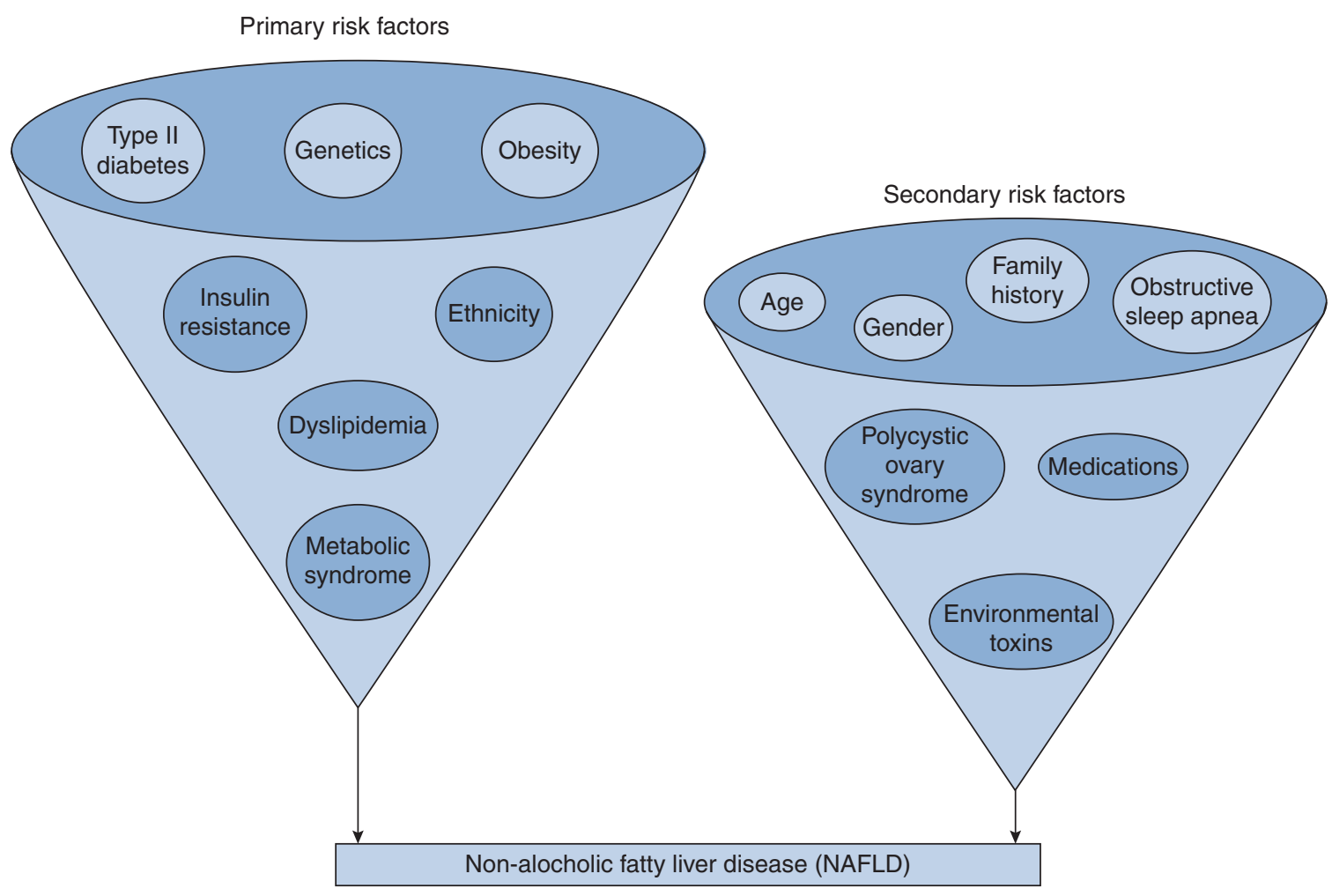

Figure 1a Risk factors for non-alcoholic fatty liver disease.

immunodeficiency virus (HIV) and hepatitis C virus (HCV) are distinct clinical entities that fall outside the spectrum of NAFLD, they do appear to exacerbate metabolic syndrome and hepatic steatosis. ${ }^{24}$

A number of medications have also been implicated and some are now considered known risk factors for NAFLD and NASH. A review of literature shows that glucocorticoids, amiodarone, tamoxifen, methotrexate, isoniazid and diltiazem and anti-retroviral therapy in HIV patients are the most common offending agents. ${ }^{12,63}$ As mentioned earlier, emerging genomics data will probably identify as yet unknown genetic risk factors for the development of NAFLD and NASH.

\section{Natural History of Non-alcoholic Fatty Liver Disease}

There are a limited number of prospective, longitudinal studies with long-term histologic follow-up of patients with NAFLD. In addition, even liver biopsies are not fully accurate in assessing the severity and progression of disease. One community-based cohort study of NAFLD subjects was noted to have a $34 \%$ increase in hazard ratio for overall mortality. ${ }^{64}$ Liver-related mortality is the third most common cause of death in large cohorts of patients with NAFLD ${ }^{64,65}$ Based on NHANES III data and other cohorts, Ong et al reported that in patients with NAFLD, liverrelated mortality seems to be higher than that in the general population, even when cofounders such as metabolic

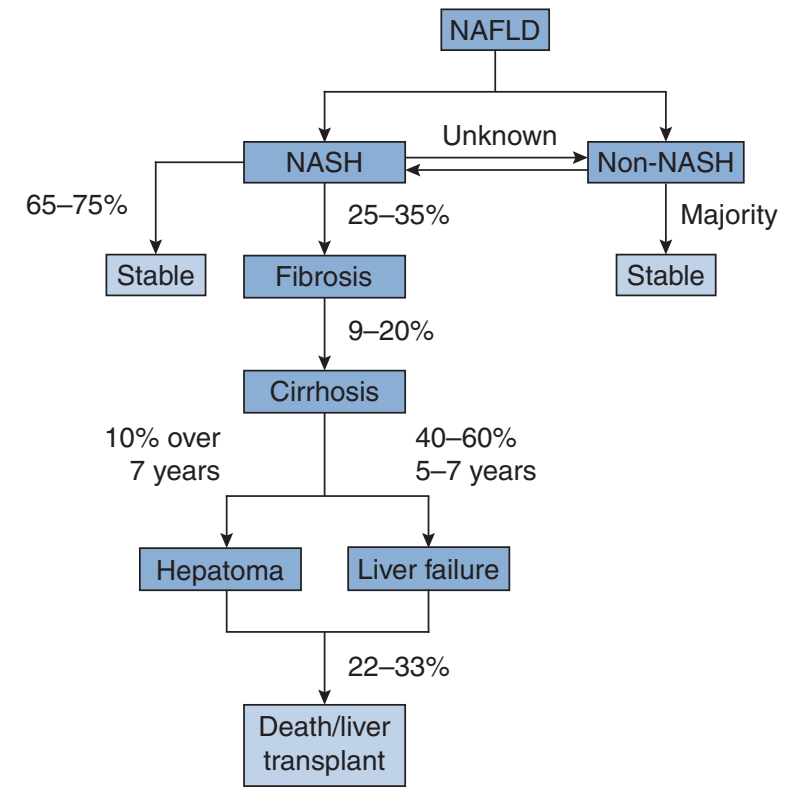

Figure 1b Natural history of NAFLD-modified from Ong, 2007. ${ }^{11}$ NAFLD: non-alcoholic fatty liver disease; NASH: non-alcoholic steatohepatitis.

factors are adjusted (Figure 1b). ${ }^{21} \mathrm{~A}$ recent meta-analysis also demonstrated that NAFLD increases the risk for allcause mortality. ${ }^{66}$ True understanding of the natural history, however, is limited by lack of long-term follow-up, difficulties with diagnosis, under-diagnosis, and other 
confounding factors, particularly the greater risk of mortality imparted by the underlying metabolic syndrome so prevalent in patients with NAFLD. Most of the data published in literature stem from large tertiary care centers that routinely perform biopsy and have greater than typical follow-up and naturally it may not be representative of the population at large.

\section{Non-alcoholic Steatohepatitis (NASH) and Non-NASH}

In looking at the natural history of NAFLD, it is extremely important to separate out NASH from non-NASH subtype of NAFLD. As previously mentioned, NAFLD has a spectrum of disease manifestation. One end is the simple steatosis, also referred to as non-alcoholic fatty liver (NAFL). Patients with simple steatosis or NAFL just have steatosis without evidence of hepatocellular injury. At the other end of the spectrum, NASH is comprised of both steatosis and hepatocellular injury and may be associated with hepatic fibrosis. Prognosis is greatly altered based on whether patients have NASH or non-NASH. It is generally accepted that patients with simple steatosis progress very slowly, if at all. Patients with NASH are more likely than non-NASH group to have progressive liver disease and can experience complications such as cirrhosis and HCC. There are probably a wide range of factors that predispose patients to progress from simple steatosis to NASH, although the exact rate of conversion is unknown. Lebovics and Rubin, in their recent review of NAFLD, cite this rate of conversion from simple steatosis to NASH at $20 \%$ over a 15 -year follow-up. ${ }^{67}$ It has also been reported in small case series that uncontrolled metabolic risk factors were the driving forces for many patients in progression from simple steatosis to NASH ${ }^{68}$ A few genomics studies, to date, have also implicated genetic factors associated with the progression of NAFLD, although these factors lack clinical application at this time. ${ }^{69}$

As noted previously, it is also interesting to note that while hepatic injury induced by NASH is similar to that seen in patients with alcoholic liver disease, NASH does appear to progress more slowly and is less severe on histology than steatohepatitis caused by alcohol. ${ }^{70,71}$ Teli et al published their data in 1995 of a cohort of 40 patients with simple steatosis that were followed for a median of 11 years, and included patients with or without risk factors for progression to NASH. ${ }^{71}$ In that series, 12 patients had abnormal liver tests but none progressed to NASH or cirrhosis and more importantly, of the 14 deceased patients; none sustained liver-related mortality. ${ }^{71}$ In another study, after 5 years of follow-up, both inflammation and ballooning, and mild fibrotic changes were seen in patients with simple steatosis. ${ }^{68}$ In a small series of $52 \mathrm{pa-}$ tients with NAFLD, who were evaluated 3 years after the initial diagnosis, among the patients that only had simple steatosis, $15 \%$ had normal liver enzymes at the 3-year follow-up, $23 \%$ remained at simple steatosis stage, $39 \%$ developed borderline NASH, and 23\% developed NASH. ${ }^{72}$ The limitation of this study was its small sample size so unlikely it can be generalized to a larger population. In another recent study of 257 NAFLD patients, based on liver biopsy results, it was noted that patients with histologic diagnosis of NASH had higher liver-related mortality and died, on average, 6 years earlier than subjects without NASH. ${ }^{73}$ Overall, despite variations in data from different centers, it does appear that simple steatosis follows a more benign course for the liver disease and is likely only slowly progressive, if at all. Non-alcoholic steatohepatitis on the other hand should be considered a progressive liver disease.

\section{Cirrhosis and Liver-related Mortality}

Over a relatively short period of follow-up, about $2 \%$ of NASH patients progressed to decompensated liver disease despite histologic progression in $10-15 \%$ of those patients. ${ }^{74,75}$ Powell et al followed up 42 patients for a median of 4.5 years, with a range of $1.5-21.5$ years. In that series, of all the patients that developed complications of cirrhosis, marked fibrosis, one case of HCC and death were all in patients that had features of NASH and not simple steatosis. ${ }^{75}$ Argo et al reviewed 221 NASH patients with a mean follow-up of 5.3 years. In that group, $37 \%$ showed progressive fibrosis. ${ }^{76}$ It is important to mention, however, that in two other studies, $18 \%$ of patients showed a drop in their fibrosis score on a mean follow-up of 5.7 years and in another, $29 \%$ of patients had regression of fibrosis on a mean follow-up of 3.2 years. ${ }^{22,77}$ Despite a lower risk of progression than alcohol-induced liver disease, NASH must be considered a risk factor for cirrhosis and increased liverrelated mortality. Just like any other patient with cirrhosis, they are at risk for development of ascites, variceal bleeding, hepatic encephalopathy, and development of HCC. In a cohort of 152 NASH subjects with cirrhosis followed over 10 years, the rate of decompensation and liverrelated deaths was $45 \%$ and $14 \%$, respectively. ${ }^{78}$ Rafiq et al, in a recent study, demonstrated a liver-related mortality of $17.5 \%$ for the NASH cohort compared with only $2.7 \%$ for the non-NASH cohort with a median follow-up of 18.5 years. ${ }^{79}$ In another study, during a mean follow-up of approximately 7 years done by Adams et al, $62 \%$ of patients sustained complications from cirrhosis and in that same group, liver-related mortality was $33 \%{ }^{22}$ Once cirrhosis develops in NASH patients, their overall 64 prognosis is negatively altered. Clinical course of patients who had presumed NASH-related cirrhosis was found by Ratziu et al to be similar to those with cirrhosis from hepatitis $\mathrm{C}$ although there were some limitations on that study. ${ }^{80}$

Additionally, it is important to note that most patients with cryptogenic cirrhosis in the USA are now considered 
Table 1 Rates of hepatocellular carcinoma cirrhotic patients due to non-alcoholic steatohepatitis vs chronic hepatitis $C$.

\begin{tabular}{lcc}
\hline & $\begin{array}{c}\text { Patients with NASH } \\
\text { or presumed NASH } \\
\text { cirrhosis (\%) }\end{array}$ & $\begin{array}{c}\text { Patients with } \\
\text { chronic hepatitis C } \\
\text { cirrhosis (\%) }\end{array}$ \\
\hline Yatsuji et $\mathrm{al}^{82}$ & 11.3 & 30.5 \\
\hline Ratziu et $\mathrm{al}^{80}$ & 8 & 18 \\
Sanyal et $\mathrm{al}^{78}$ & 6.7 & 17 \\
\hline
\end{tabular}

Note that the duration of follow-up was variable in the three studies but all show a higher overall rate with hepatitis C vs NASH cirrhosis. NASH: non-alcoholic steatohepatitis.

to have had burned-out NASH. These patients have high prevalence of conditions associated with metabolic syndrome and NASH can re-occur after LT. $^{24}$

\section{Hepatocellular Carcinoma}

There is some uncertainty about the risk of HCC in NASH or NAFLD patients. It is accepted by most experts that non-NASH patients with steatosis alone are not at risk for HCC. Of the NASH cohorts, patients with cirrhosis are thought to be at risk for development of HCC. The 2005 American Association for the Study of Liver Diseases (AASLD) guidelines do recommend that all patients who are at high-risk, such as those with cirrhosis of any cause or certain noncirrhotic hepatitis B carriers, should be enrolled in surveillance programs for screening for HCC. ${ }^{81}$ Hence, NASH-related cirrhotic patients certainly fall within these guidelines. Yatsuji et al put the incidence of HCC at $11.3 \%$ in 5 years for patients with cirrhosis secondary to NASH which is notable for a lower overall risk than patients with hepatitis C-related cirrhosis. ${ }^{82}$ Other experts have put the annual incidence of HCC in subjects with NASH-related cirrhosis to be approximately $2.6 \%{ }^{78}$ Given the body habitus (visceral obesity) and relative contraindication for LT (obesity, diabetes, coronary artery disease), a large number of patients with NASH do not get screening for HCC. In fact, these patients may present with large tumors which may not be amenable to treatment. At this time, the exact pathogenesis of NASH-related HCC remains unclear although experts do agree that age and advanced fibrosis are established risk factors for HCC in NASH. In looking at the published data, while there are variations from study to study on the overall rate of NASH-related HCC, there is consistent evidence that the risk of HCC is greater with hepatitis C-related cirrhosis.

\section{Liver Transplantation}

Cirrhotic NASH patients with decompensated cirrhosis or hepatomas can be considered for LT. In reality, this is probably complicated by other underlying risk factors in that patient population with high incidence of diabetes and cardiovascular risk factors. Based on published literature, the outcomes of LT in cirrhotic NASH patients are similar to those with other etiologies of liver diseases undergoing transplantation. The reported 5-year survival has ranged from $71 \%$ to $75 \%$ and recurrence is also common in the post-transplant liver occurring in $20-33 \%$ of cases with peri-portal fibrosis seen in $18 \%$ of patients by 18 months. ${ }^{83,84}$

\section{Cardiovascular Diseases}

Any discussion of NAFLD is not complete without looking at the cardiovascular disease (CVD) risk in that patient population. Given the fact that NAFLD and CVDs share similar risk factors of obesity, type II diabetes, insulin resistance, dyslipidemia, and metabolic syndrome, it is not surprising that NAFLD patients have a higher incidence of cardiovascular events. However, there are data suggesting that in patients with NAFLD, there is still a higher incidence of CVDs compared with matched controls independent of other cardiovascular risk factors. ${ }^{14,85}$ Hence, in looking at the natural history of NAFLD, it is important to keep this issue in mind. The NAFLD and its manifestations are not only related to increase liver-related mortality but also related to cardiovascular morbidity and mortality. Some authors have noted that CVD is a leading cause of death in cohorts of patients who have NAFLD. ${ }^{22,40}$ It is possible that in addition to the shared underlying risk factors for atherosclerosis, there may be an increased risk by virtue of NAFLD itself.

\section{Diabetes}

We have previously stressed the importance of diabetes type II and its surrogate, insulin resistance as key factors in the pathogenesis of NAFLD and important risk factor for NASH. However, it is important to remember that NAFLD may precede and increase the risk of diabetes type II as well. Although more research is needed in this area, cohort studies have shed some light on this. Even after adjusting for potential confounding factors, it was noted in two separate studies in Korea and Japan that ultrasound diagnosed NAFLD portends an increased risk of developing diabetes type II (odds ratio $1.5-5.5$ ) over $4-5$ years. ${ }^{86,87}$

\section{Resolution of Non-alcoholic Fatty Liver Disease}

There is paucity of data in the literature regarding true resolution of NAFLD. This is further confounded by lack of long-term follow-up and sparse biopsy data on large population-based studies. The most compelling data to date come from the bariatric surgery population. Hepatic infiltration with fat was reduced in 65 of 91 patients in one study of patients biopsied at the time of bariatric surgery and at follow-up. ${ }^{88}$ In another study of 90 biopsied bariatric surgery patients, $18 \%$ remained at the same degree of steatosis, $28 \%$ showed improvement in steatosis 
and $54 \%$ reverted to normal hepatic tissue by the time of second biopsy. ${ }^{89}$

\section{CONCLUSION}

Over the past 20 years, we have had a much better understanding of NAFLD and NASH. On-going research and population data will give us additional information. Nonalcoholic fatty liver disease and NASH will be a significant burden to the Western society and to the world as obesity rates and its associated comorbid conditions such as diabetes, dyslipidemia, and metabolic syndromes rise across the world. We know based on data previously discussed that as populations gain weight, NAFLD will follow. There are additional risk factors of PCOS, sleep apnea, plethora of medications, age in addition to the well-established ones that will surely increase the disease burden of NAFLD and its protean manifestations. Although simple steatosis subtype of NAFLD generally carries a good prognosis from a liver standpoint and is considered to be nonprogressive or slowly progressive, the same is not true of NASH. Nonalcoholic steatohepatitis can lead to cirrhosis and its complications. In the US alone, experts and epidemiologic data suggest that at least one-third of the population is obese and most likely has NAFLD and approximately $2-5 \%$ have NASH. ${ }^{11}$ This incidence may indeed be even higher given the lack of biopsy data and lack of accurate non-invasive biomarkers for NAFLD. There are many unanswered questions in many areas as identified above and on-going large-scale, long-term prospective studies looking at outcomes will give more definitive answers.

\section{CONFLICTS OF INTEREST}

All authors have none to declare.

\section{REFERENCES}

1. Chitturi S, Farrell GC, Hashimoto E, Saibara T, Lau GK, Sollano JD. Non-alcoholic fatty liver disease in the Asia-Pacific region: definitions and overview of proposed guidelines. Asia-Pacific Working Party on NAFLD. J Gastroenterol Hepatol 2007;22:778.

2. Brunt EM, Janney CG, Di Bisceglie AM, Neuschwander-Tetri BA, Bacon BR. Nonalcoholic steatohepatitis: a proposal for grading and staging the histological lesions. Am J Gastroenterol 1999;94: 2467-74.

3. American Gastroenterological Association (AGA) medical position Statement. Non-alcoholic fatty liver disease. Gastroenterology 2002;123:1702-4.

4. Whalley S, Puvanachandra P, Desia A, Kennedy H. Hepatology outpatient service provision in secondary care: a study of liver disease incidence and resource costs. Clin Med 2007;7:119-24.

5. Suzuki A, Angulo P, Lymp J, et al. Chronological development of elevated aminotransferases in a nonalcoholic population. Hepatology 2005;41:64-71.

6. Hamaguchi M, Kojima T, Takeda N, et al. The metabolic syndrome as a predictor of non-alcoholic fatty liver disease. Ann Intern Med 2005;143:722-8.

7. Browning JD, Szczepaniak LS, Dobbins R, et al. Prevalence of hepatic steatosis in an urban population in the United States: impact of ethnicity. Hepatology 2004;40:1387-95.
8. Mohanty SR, Troy TN, Huo D, O'Brien BL, Jensen DM, Hart J. Influence of ethnicity on histological differences in non-alcoholic fatty liver disease. J Hepatol 2009;50:797-804.

9. Lee JY, Kim KM, Lee SG, et al. Prevalence and risk factors of non-alcoholic fatty liver disease in potential living liver donors in Korea: a review of 589 consecutive liver biopsies in a single center. $J$ Hepatol 2007;47:239-44.

10. Marcos A, Fisher RA, Ham JM, et al. Selection and outcome of living donors for adult to adult right lobe transplantation. Transplantation 2000;69:2410-5.

11. Ong JP, Younossi ZM. Epidemiology and natural history of NAFLD and NASH. Clin Liver Dis 2007;11:1-16.

12. Kopec KL, Burns D. Nonalcoholic fatty liver disease. a review of the spectrum of disease, diagnosis, and therapy. Nutr Clin Pract 2011;26:565-76.

13. Ong JP, Elariny $\mathrm{H}$, Collantes $\mathrm{R}$, et al. Predictors of nonalcoholic steatohepatitis and advanced fibrosis in morbidly obese patients. Obes Surg 2005;15:310-5.

14. Hamaguchi M, Kojima T, Takeda N, et al. Nonalcoholic fatty liver disease is a novel predictor of cardiovascular disease. World $J$ Gastroenterol 2007;13:1579-84.

15. Chalasani N, Younossi Z, Lavine JE, et al. The diagnosis and management of non-alcoholic fatty liver disease: Practice guideline by the american association for the study of liver diseases, American College of Gastroenterology, and the American Gastroenterological Association. Hepatology 2012.

16. Younossi ZM, Stepanova M, Afendy M, et al. Changes in the prevalence of the most common causes of chronic liver diseases in the United States from 1988 to 2008. Clinical Gastroenterol Hepatol 2011;9:524-30.

17. Fan JG, Zhu J, Li XJ, et al. Prevalence of and risk factors for fatty liver in a general population of Shanghai, China. $J$ Hepatol 2005; 43:508-14.

18. Ruhl CE, Everhart JE. Determinants of the association of overweight with elevated serum alanine aminotransferase activity in the United States. Gastroenterology 2003;124:71-9.

19. Frith J, Day CP, Henderson E, Burt AD, Newton JL. Non-alcoholic fatty liver disease in older people. Gerontology 2009;55: 607-13.

20. Chen $\mathrm{CH}$, Huang $\mathrm{MH}$, Yang JC, et al. Prevalence and etiology of elevated serum alanine aminotransferase level in an adult population in Taiwan. J Gastroenterol Hepatol 2007;22:1482-9.

21. Ong JP, Pitts A, Younossi ZM. Increased overall mortality and liverrelated mortality in non-alcoholic fatty liver disease. J Hepatol 2008;49:608-12.

22. Hossain N, Afendy A, Stepanova M, et al. Independent predictors of fibrosis in patients with non-alcoholic fatty liver disease. Clin Gastroenterol Hepatol 2009;7:1224-9.

23. Kagansky N, Levy S, Keter D, et al. Non-alcoholic fatty liver disease-a common and benign finding in octogenarian patients. Liver Int 2004;24:588-94.

24. Vernon G, Baranova A, Younossi ZM. Systematic review: the epidemiology and natural history of non-alcoholic fatty liver disease and non-alcoholic steatohepatitis in adults. Aliment Pharmacol Ther 2011;34:274-85.

25. Reid A. Nonalcoholic steatohepatitis. Gastroenterology 2001;121: 710-23.

26. Chen ZW, Chen LY, Dai HL, Chen JH, Fang LZ. Relationship between alanine aminotransferase levels and metabolic syndrome in nonalcoholic fatty liver disease. J Zhejiang Univ Sci B 2008; 9:616-22.

27. Arun J, Clements RH, Lazenby AJ, Leeth RR, Abrams GA. The prevalence of nonalcoholic steatohepatitis is greater in morbidly obese men compared to women. Obes Surg 2006;16:1351.

28. Williams CD, Stengel J, Asike MI, et al. Prevalence of nonalcoholic fatty liver disease and nonalcoholic steatohepatitis among a 
largely middle-aged population utilizing ultrasound and liver biopsy: a prospective study. Gastroenterology 2011;140:124.

29. Wagenknecht LE, Scherzinger AL, Stamm ER, et al. Correlates and heritability of nonalcoholic fatty liver disease in a minority cohort. Obesity 2009;17:1240-6.

30. Romeo S, Kozlitina J, Xing C, et al. Genetic variation in PNPLA3 confers susceptibility to nonalcoholic fatty liver disease. Nat Genet 2008;40:1461-5.

31. Valenti L, Al-Serri A, Daly AK, et al. Homozygosity for the patatinlike phospholipase-3/adiponutrin I148M polymorphism influences liver fibrosis in patients with nonalcoholic fatty liver disease. Hepatology 2010;51:1209-17.

32. He S, McPhaul C, Li JZ, et al. A sequence variation (I148M) in PNPLA3 associated with nonalcoholic fatty liver disease disrupts triglyceride hydrolysis. J Biol Chem 2010;285:6706-15.

33. Petersen KF, Dufour S, Hariri A, et al. Apolipoprotein C3 gene variants in nonalcoholic fatty liver disease. N Engl J Med 2010;362: 1082-9.

34. Speliotes EK, Yerges-Armstrong LM, Wu J, et al. Genome-wide association analysis identifies variants associated with nonalcoholic fatty liver disease that have distinct effects on metabolic traits. PLoS Genet 2011; 7:e1001324.

35. Leite NC, Salles GF, Araujo AL, Villela-Nogueira CA, Cardoso CR. Prevalence and associated factors of non-alcoholic fatty liver disease in patients with type-2 diabetes mellitus. Liver Int 2009; 29:113-9.

36. Prashanth M, Ganesh HK, Vima MV, et al. Prevalence of nonalcoholic fatty liver disease in patients with type 2 diabetes mellitus. J Assoc Physicians India 2009;57:205-10.

37. Adams LA, Angulo P, Lindor KD. Nonalcoholic fatty liver disease. CMAJ 2005;172:899-905.

38. Younossi ZM, Gramlich T, Matteoni CA, et al. Nonalcoholic fatty liver disease in patients with type 2 diabetes. Clin Gastroenterol Hepatol 2004;2:262-5.

39. Stepanova M, Rafiq N, Younossi ZM. Components of metabolic syndrome are independent predictors of mortality in patients with chronic liver disease: a population-based study. Gut 2010;59: 1410-5.

40. Matteoni CA, Younossi ZM, Gramlich T, Boparai N, Liu YC, McCullough AJ. Nonalcoholic fatty liver disease: a spectrum of clinical and pathological severity. Gastroenterology 1999;116:1413-9.

41. Angelico F, Del Ben M, Conti R, et al. Non-alcoholic fatty liver syndrome: a hepatic consequence of common metabolic diseases. J Gastroenterol Hepatol 2003;18:588-94.

42. Boza C, Riquelme A, Ibañez L, et al. Predictors of nonalcoholic steatohepatitis (NASH) in obese patients undergoing gastric bypass. Obes Surg 2005;15:1148-53.

43. Abrams GA, Kunde SS, Lazenby AJ, Clements RH. Portal fibrosis and hepatic steatosis in morbidly obese subjects: a spectrum of nonalcoholic fatty liver disease. Hepatology 2004;40:475-83.

44. Machado M, Marques-Vidal P, Cortez-Pinto H. Hepatic histology in obese patients undergoing bariatric surgery. J Hepatol 2006;45: 600-6.

45. Colicchio P, Tarantino G, del Genio F, et al. Non-alcoholic fatty liver disease in young adult severely obese non-diabetic patients in South Italy. Ann Nutr Metab 2005;49:289-95.

46. Beymer C, Kowdley KV, Larson A, Edmonson P, Dellinger EP, Flum DR. Prevalence and predictors of asymptomatic liver disease in patients undergoing gastric bypass surgery. Arch Surg 2003; 138:1240-4.

47. Bellentani S, Saccoccio G, Masutti F, et al. Prevalence of and risk factors for hepatic steatosis in Northern Italy. Ann Intern Med 2000;132:112-7.

48. Dolce CJ, Russo M, Keller JE, et al. Does liver appearance predict histopathologic findings: prospective analysis of routine liver biopsies during bariatric surgery. Surg Obes Relat Dis 2009;5:323-8.
49. Gholam PM, Flancbaum L, Machan JT, Charney DA, Kotler DP. Nonalcoholic fatty liver disease in severely obese subjects. Am J Gastroenterol 2007;102:399-408.

50. Harnois F, Msika S, Sabaté JM, et al. Prevalence and predictive factors of non-alcoholic steatohepatitis (NASH) in morbidly obese patients undergoing bariatric surgery. Obes Surg 2006;16: 183-8.

51. Spaulding L, Trainer T, Janiec D. Prevalence of non-alcoholic steatohepatitis in morbidly obese subjects undergoing gastric bypass. Obes Surg 2003;13:347-9.

52. Farrell GC, Larter CZ. Nonalcoholic fatty liver disease: from steatosis to cirrhosis. Hepatology 2006;43:s99-112.

53. Ehrmann DA. Polycystic ovary syndrome. N Engl J Med 2005;352: 1223-36.

54. Setji TL, Brown AJ. Polycystic ovary syndrome: diagnosis and treatment. Am J Med 2007;120:128-32.

55. Welt CK, Gudmundsson JA, Arason G, et al. Characterizing discrete subsets of polycystic ovary syndrome as defined by the Rotterdam criteria: the impact of weight on phenotype and metabolic features. J Clin Endocrinol Metab 2006;91:4842-8.

56. Cerda C, Perez-Ayuso RM, Riquelme A, et al. Nonalcoholic fatty liver disease in women with polycystic ovary syndrome. $J$ Hepatol 2007;47:412-7.

57. Hossain N, Stepanova M, Afendy A, et al. Non-alcoholic steatohepatitis (NASH) in patients with polycystic ovarian syndrome (PCOS). Scand J Gastroenterol 2011;46:479-84.

58. Ahmed MH, Byrne CD, Obstructive sleep apnea syndrome and fatty liver: association or causal link? World J Gastroenterol 2010; 16:4243-52.

59. Vgontzas AN, Bixler EO, Chrousos GP. Sleep apnea is a manifestation of the metabolic syndrome. Sleep Med Rev 2005;9: 211-24.

60. Keijer J, van Schothorst EM. Adipose tissue failure and mitochondria as a possible target for improvement by bioactive food components. Curr Opin Lipidol 2008;19:4-10.

61. Mishra P, Nugent C, Afendy A, et al. Apnoeic-hypopnoeic episodes during obstructive sleep apnoea are associated with histological nonalcoholic steatohepatitis. Liver Int 2008;28:1080-6.

62. Requarth JA, Burchard KW, Colacchio TA, et al. Long term morbidity following jejunoileal bypass: the continuing potential need for surgical reversal. Arch Surg 1995:130:318-25.

63. Grundy SM, Brewere HB Jr, Celleman JI, Smith SC Jr, Lenfant C. Definition of metabolic syndrome: report of the National Heart Lung and Blood Institute/American Heart Association Conference on scientific issues related to definition. Circulation 2004;109: 433-8.

64. Adams LA, Lymp JF, St Sauver J, et al. The natural history of nonalcoholic fatty liver disease: a population-based cohort study. Gastroenterology 2005;129:113-21.

65. Soderberg C, Stal P, Askling J, et al. Decreased survival of subjects with elevated liver function tests during a 28 year follow-up. Hepatology 2010;51:595-602.

66. Musso G, Gambino R, Cassader M, Pagano G. Meta-analysis: natural history of non-alcoholic fatty liver disease and diagnostic accuracy of non-invasive tests for liver disease severity. Ann Med 2011;43:617-49.

67. Lebovics E, Rubin J. Non-alcoholic fatty liver disease (NAFLD): why you should care, when you should worry, what you should do. Diabetes Metab Res Rev 2011;27:419-24.

68. Pais R, Pascale A, Fedchuck L, Charlotte F, Poynard T, Ratziu V. Progression from isolated steatosis to steatohepatitis and fibrosis in nonalcoholic fatty liver disease. Clin Res Hepatol Gastroenterol 2011;35:23-8.

69. Hernaez R. Genetic factors associated with the presence and progression of nonalcoholic fatty liver disease: a narrative review. Gastroenterol Hepatol 2011 [Epub ahead of print]. 
70. Ludwig J, Viggiano TR, McGill DB, Oh BJ. Nonalcoholic steatohepatitis: Mayo Clinic experiences with a hitherto unnamed disease. Mayo Clin Proc 1980;55:434-8.

71. Teli MR, James OF, Burt AD, Bennett MK, Day CP. The natural history of nonalcoholic fatty liver: a follow-up study. Hepatology 1995;22:1714-9.

72. Wong VW, Wong GL, Choi PC, et al. Disease progression of nonalcoholic fatty liver disease: a prospective study with paired liver biopsies at 3 years. Gut 2010;59:969-74.

73. Younossi ZM, Stepanova M, Rafiq N, et al. Pathologic criteria for nonalcoholic steatohepatitis: interprotocol agreement and ability to predict liver-related mortality. Hepatology 2011;53: 1874-82.

74. Lee RG. Nonalcoholic steatohepatitis: a study of 49 patients. Hum Pathol 1989;20:594-8.

75. Powell EE, Cooksley WG, Hanson R, et al. The natural history of nonalcoholic steatohepatitis: a follow-up study of forty-two patients for up to 21 years. Hepatology 1990;11:74-80.

76. Argo CK, Northup PG, Al-Osaimi AM, Caldwell SH. Systematic review of risk factors for fibrosis progression in non-alcoholic steatohepatitis. J Hepatol 2009;51:371-9.

77. Harrison SA, Torgerson S, Hayashi PH. The natural history of nonalcoholic fatty liver disease: a clinical histopathological study. Am J Gastroenterol 2003;98:2042-7.

78. Sanyal AJ, Banas C, Sargent C, et al. Similarities and differences in outcomes of cirrhosis due to nonalcoholic steatohepatitis and hepatitis C. Hepatology 2006;43:682-9.

79. Rafiq N, Bai C, Fang Y, et al. Long-term follow-up of patients with nonalcoholic fatty liver. Clin Gastroenterol Hepatol 2009;7:234-8.

80. Ratziu V, Bonyhay L, Di Martino V, et al. Survival, liver failure, and hepatocellular carcinoma is obesity-related cryptogenic cirrhosis. Hepatology 2002;35:1485-93.
81. Bruix J, Sherman M. Practice Guidelines Committee, American Association for the Study of Liver Diseases. Management of hepatocellular carcinoma. Hepatology 2005;42:1208-36.

82. Yatsuji S, Hashimoto E, Tobari M, et al. Clinical features and outcomes of cirrhosis due to non-alcoholic steatohepatitis compared with cirrhosis caused by chronic hepatitis C. J Gastoenterol Hepatol 2009;24:248-54.

83. Bhagat V, Mindikoglu AL, Nudo CG, et al. Outcomes of liver transplantation in patients with cirrhosis due to nonalcoholic steatohepatitis versus patients with cirrhosis due to alcoholic liver disease. Liver Transpl 2009;15:1814-20.

84. Yalamanchili K, Saadeh S, Klintmalm GB, et al. Nonalcoholic fatty liver disease after liver transplantation for cryptogenic cirrhosis or nonalcoholic liver disease. Liver Transpl 2010;16: 431-9.

85. Targher G, Bertolini L, Rodella S, et al. Nonalcoholic fatty liver disease is independently associated with an increased incidence of cardiovascular events in type 2 diabetic patients. Diabetes Care 2007;30:2119-21.

86. Kim CH, Park JY, Lee KU, et al. Fatty liver is an independent risk factor for the development of Type II diabetes in Korean adults. Diabetes Med 2008;25:476-81.

87. Yamada T, Fukatsu M, Suzuki S, et al. Fatty liver predicts impaired fasting glucose and type 2 diabetes mellitus in Japanese undergoing a health check-up. J Gastroenterol Hepatol 2010;25: 352-6.

88. Silverman EM, Sapala JA, Appelman HD. Regression of hepatic steatosis in morbidly obese persons after gastric bypass. Am J Clin Pathol 1995;104:23-31.

89. Mottin CC, Moretto M, Padoin AV, et al. Histological behavior of hepatic steatosis in morbidly obese patients after weight lass induced by bariatric surgery. Obes Surg 2005;15:788-93. 